Article

\title{
Fast Reconfigurable SOA-Based Wavelength Conversion of Advanced Modulation Format Data ${ }^{\dagger}$
}

\author{
Yi Lin ${ }^{1, *}$, Aravind P. Anthur ${ }^{1}$, Sean P. Ó Dúill ${ }^{1}$ (i), Fan Liu ${ }^{2}$, Yonglin Yu ${ }^{2}$ and Liam P. Barry ${ }^{1}$ \\ 1 School of Electronic Engineering, Dublin City University, Dublin 9, Ireland; aravind.anthur@dcu.ie (A.P.A.); \\ sean.oduill@dcu.ie (S.P.ÓD.); liam.barry@dcu.ie (L.P.B.) \\ 2 Wuhan National Laboratory for Optoelectronics, Huazhong University of Science and Technology, \\ Wuhan 430073, China; fliu@hust.edu.cn (F.L.); yonglinyu@mail.hust.edu.cn (Y.Y.) \\ * Correspondence: yi.lin6@mail.dcu.ie; Tel.: +353-1-700-5883 \\ + This paper is an extended version of paper published in the The Optical Fiber Communication Conference \\ and Exhibition, OFC 2017 held in Los Angeles, 19-23 March 2017.
}

Received: 31 August 2017; Accepted: 3 October 2017; Published: 10 October 2017

\begin{abstract}
We theoretically analyze the phase noise transfer issue between the pump and the wavelength-converted idler for a nondegenerate four-wave mixing (FWM) scheme, as well as study the vector theory in nonlinear semiconductor optical amplifiers (SOAs), in order to design a polarization-insensitive wavelength conversion system employing dual co-polarized pumps. A tunable sampled grating distributed Bragg reflector (SG-DBR) pump laser has been utilized to enable fast wavelength conversion in the sub-microsecond timescale. By using the detailed characterization of the SGDBR laser, we discuss the phase noise performance of the SGDBR laser. Finally, we present a reconfigurable SOA-based all-optical wavelength converter using the fast switching SGDBR tunable laser as one of the pump sources and experimentally study the wavelength conversion of the single polarization quadrature phase shift keying (QPSK) and polarization multiplexed (Pol-Mux) QPSK signals at 12.5-Gbaud. A wide tuning range ( $>10 \mathrm{~nm}$ ) and less than $50 \mathrm{~ns}$ and $160 \mathrm{~ns}$ reconfiguration time have been achieved for the wavelength conversion system for QPSK and PM-QPSK signals, respectively. The performance under the switching environment after the required reconfiguration time is the same as the static case when the wavelengths are fixed.
\end{abstract}

Keywords: all-optical networks; wavelength conversion devices; tunable lasers

\section{Introduction}

The massive growth in the demand for bandwidth for multimedia services and interactive networks is shaping a new era for today's communication networks. Future networks need to offer bandwidth-hungry applications like telemedicine, IP-TV, virtual reality gaming, video-on-demand, and high-speed internet access, combined with guaranteed Quality of Service [1]. In optical networks that employ wavelength division multiplexed (WDM), the use of optical switching technologies on a burst or packet level, combined with advanced modulation formats, would achieve greater spectral efficiency and utilize the existing bandwidth more efficiently. All-optical wavelength converters which typically comprise of tunable pump lasers, nonlinear media, and the tunable optical filter, are expected to be one of the key components in these broadband networks. They can be used to interface different networks and potentially increase the capacity of a communication system [2,3]. The wavelength converters can be also used at the network nodes to avoid contention and to dynamically allocate wavelengths to ensure optimum use of fiber bandwidth [4].

Recently, significant work has been undertaken on wavelength conversion based on four-wave mixing (FWM) by using different nonlinear devices such as nonlinear semiconductor optical amplifiers (SOAs) [5], highly nonlinear fiber (HNLF) [6-8], periodically poled Lithium Niobate (PPLN) [9], and 
nonlinear waveguides [10-12]. Nonlinear SOAs give the best conversion efficiency among these nonlinear devices because they are active devices [13]. To perform the wavelength conversion of data signals where the information is encoded onto the phase and amplitude of the optical carrier, e.g., quadrature phase shift keying (QPSK) and 16-ary state quadrature amplitude modulation (16-QAM), the wavelength conversion process must preserve the amplitude and phase information. Therefore, FWM is required as the wavelength conversion process because FWM preserves the amplitude and phase information of the input signal. In addition, FWM is transparent to the different modulation formats where the information can be encoded in the amplitude, phase, and polarization of the optical carrier [14], and is also transparent to the signal baud rate (the wavelength conversion of the on-off keying (OOK) signal at 100 Gbaud having been demonstrated in [15]). Recently, a lot of work has been done on the wavelength conversion of data signals employing different modulated formats including (Differential) QPSK, 8-Phase Shift Keying (PSK), 16-QAM, and 64-QAM based on FWM in SOAs using single pump and dual pump wavelength conversion systems [16-27].

In-addition to phase and amplitude, optical communication networks are employing polarization multiplexed (Pol-Mux) modulation formats to achieve twice the spectral efficiency by encoding data on two orthogonal polarizations of the light. When data is present in two orthogonal polarizations, it is very important to ensure that FWM-based wavelength converters are transparent to Pol-Mux modulation formats. Vector theory of FWM in nonlinear media is well understood and utilized for achieving polarization-insensitive wavelength conversion [28]. Vector theory was studied and verified in nonlinear SOAs and this was utilized to design a polarization-insensitive wavelength conversion scheme for Pol-Mux modulation formats utilizing dual co-polarized pumps in our earlier work [29]. Utilizing the understanding from these phase noise and polarization studies, we designed the most efficient and impairment-free wavelength converter based on FWM. We use this design to study the dynamic characteristics of the FWM-based wavelength converter and present the results, when the data is communicated in bursts/packets.

One important issue that needs to be considered when using FWM for all-optical wavelength conversion of data signals, where the information is encoded onto the phase of the optical carrier, is the phase noise transfer issue between the pump and the converted idler. In [30], a simple relationship between the linewidth of the signal, pump, and converted idler in the FWM process was found by theoretical analysis and verified experimentally. For the case of the single pump FWM scheme shown in Figure 1a, the linewidth of the converted idler is the linewidth of the signal plus four times pump linewidth, and for the case of the dual pump FWM scheme shown in Figure 1b, the linewidth of the converted idler is equal to the summed linewidths of the signal and the dual pumps. The phase noise transfer problem will cause a significant effect for the FWM-based wavelength conversion of data with phase encoding, as it becomes more difficult to recover the data with the induced uncertainty in the phase of the wavelength converted idler. Thus, the linewidth is an important parameter for the pump laser to be employed in the FWM-based wavelength conversion system.

An example of a typical all-optical packet-switched network which employs wavelength conversion is illustrated in Figure 1c. IP packets enter the network through the edge router where they are retransmitted on a new wavelength to avoid contention [31]. By employing the all-optical wavelength converter in this network, it can enable rapid routing of the same wavelength channels from any direction to any direction, and easily avoid the contention in which different packets with the same wavelengths are trying to leave the edge router. The wavelength converter consists of fast tuning tunable lasers as the pumps, an SOA, and a tunable optical filter. The wavelength of the wavelength-converted signal through FWM can be precisely altered by tuning the wavelength of the pump sources, and after the nonlinear wavelength conversion process in the SOA, the converted signal is then filtered out by using a tunable optical filter and sent to the next network node. The switching speed of this SOA-based wavelength converter mainly depends on the tunable pump lasers and the tunable optical filter [32], but in this work we focus on the effect of the tunable laser. The laser wavelength tuning speed can be as low as several ns for various tunable laser designs such as the 
sampled grating distributed Bragg reflector (SG-DBR) laser [33], the digital super-mode distributed Bragg grating (DS-DBR) laser [34], and the modulated grating Y-branch (MGY) laser [35]. These lasers can also achieve a wide tuning range $(>45 \mathrm{~nm}$ ), a high side mode suppression ratio (SMSR $>35 \mathrm{~dB}$ ), and large output power ( $>10 \mathrm{dBm})$. All the components in the wavelength converter (lasers and SOA) can be integrated and these results motivate the construction of a compact, optically-integrated, and rapidly reconfigurable all-optical wavelength converter.

Most of the previous research on wavelength conversion is undertaken by using static, fixed-wavelength lasers. However, wavelength converters with rapid, dynamic wavelength re-configurability would be required to bypass the power-hungry electronic switches with high latency in the switching nodes of future transparent optical networks. The main focus of this paper is thus to develop a wavelength converter comprised of an SOA as the nonlinear element and a fast-switching SG-DBR tunable laser as one of the pump sources. In Section 2, we initially discuss the theory of the phase noise transfer issue in FWM and the dual co-polarized pumping scheme. In Section 3, using the detailed characterization of the SGDBR laser, we discuss the phase noise of the SGDBR. In Section 4, we provide a complete set of experimental results for single polarization QPSK [36] and experimentally demonstrate rapid wavelength conversion of a PM-QPSK signal with the switching time of tens of nanoseconds using a fast-switching, tunable laser as one of the pumps in a dual wavelength pumping scheme. After the reconfiguration time, the performance under a switching scenario is the same as the case when the wavelengths are held static. The experimental results indicate that the incoming signal can be precisely and quickly converted to the required wavelength channel on a nanosecond timescale.

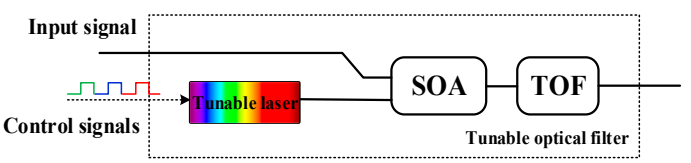

(a)

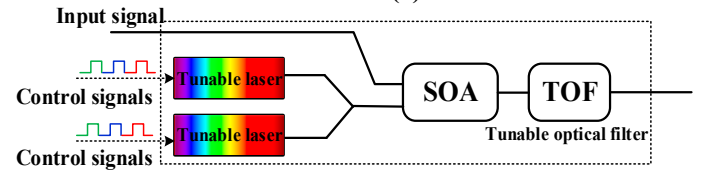

(b)

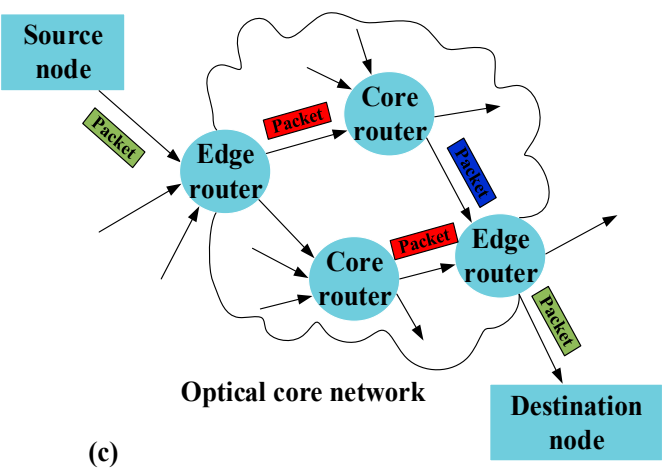

(c)

Figure 1. (a) A single pump semiconductor optical amplifier (SOA)-based wavelength converter employing one fast tunable pump laser. (b) A dual-pump SOA-based wavelength converter employing fast tunable pump lasers where the output wavelengths can be chosen by appropriately selecting the wavelength of the tunable lasers. The wavelengths of the tunable lasers are adjusted by the control signals to the SG-DBR devices. (c) Schematic of an all-optical packet-switched network.

\section{Theory}

\subsection{Phase Noise and FWM}

Consider two pumps with electric field intensities given by $\vec{E}_{p 2}$ and $\vec{E}_{p 1}$ mixing with a signal having an electrical field intensity of $\vec{E}_{s}$, generating an idler through FWM with a field intensity of $E_{i}$. Let the frequencies of the mixing pumps and the signal be represented as $\omega_{p 1}, \omega_{p 2}$, and $\omega_{s}$, respectively. Let the phase of the mixing pumps and the signal be represented as $\phi_{p 1}, \phi_{p 2}$, and $\phi_{s}$ respectively. The frequency and the phase of the idler are related to the mixing frequencies by,

$$
\begin{gathered}
\omega_{i}=\omega_{p 2}-\omega_{p 1}+\omega_{s} \\
\phi_{i}=\phi_{p 2}-\phi_{p 1}+\phi_{s}
\end{gathered}
$$


Unless both pumps have correlated phase noise, the phase noise of the idler is related to the phase noise of the mixing frequencies by the following relationship when the phase noise of the mixing frequencies is uncorrelated [16,30],

$$
\Delta \sigma_{i}^{2}=\Delta \sigma_{p 1}^{2}+\Delta \sigma_{p 2}^{2}+\Delta \sigma_{s}^{2}
$$

where $\Delta \sigma^{2}$ represents the variance of the phase noise. For white frequency phase noise, the linewidth (represented by $\Delta \omega$ ) and the phase error variance are linearly related [37]. Therefore, the linewidth of the idler due to white phase noise is related to the linewidth of the mixing pumps and the signal by,

$$
\Delta \omega_{i}=\Delta \omega_{p 1}+\Delta \omega_{p 2}+\Delta \omega_{s}
$$

Thus it can be observed from Equation (4) that the phase noise of the idler will be the sum of the phase noise of the mixing frequencies.

\subsection{FWM with Dual Co-Polarized Pumps}

We next look at the vector theory of FWM in nonlinear SOA. The states of polarization of the two co-polarized pumps and signal are shown in Figure 2a. Pump1 and pump2 are co-polarized. For simplicity, we consider a linearly polarized input signal at an arbitrary angle $\theta$ with respect to the pumps. The output spectrum of this FWM scheme is given in Figure $2 b$. The converted idlers generated by the beating between the dual co-polarized pumps have optical frequencies $\omega_{p 1}-\omega_{p 2}+\omega_{s}$ and $\omega_{p 2}-\omega_{p 1}+\omega_{s}$. We show that the FWM wavelength conversion scheme preserves the polarization properties of the signal within the idler at frequency $\omega_{p 2}-\omega_{p 1}+\omega_{s}$. The electric field of the two co-polarized pumps $\left(\vec{E}_{p 1}\right.$ and $\left.\vec{E}_{p 2}\right)$ and signal $\left(\vec{E}_{s}\right)$ are given by,

$$
\begin{gathered}
\vec{E}_{p 1}=\mathrm{A}_{p 1} \exp \left(j\left(\omega_{p 1} t+\phi_{p 1}\right)\right) \vec{x} \\
\vec{E}_{p 2}=\mathrm{A}_{p 2} \exp \left(j\left(\omega_{p 2} t+\phi_{p 2}\right)\right) \vec{x} \\
\vec{E}_{s}=\mathrm{A}_{s} \exp \left(j\left(\omega_{s} t+\phi_{s}\right)\right)[\cos (\theta) \vec{x}+\sin (\theta) \vec{y}]
\end{gathered}
$$

where $\mathrm{A}_{p 1}, \mathrm{~A}_{p 2}$, and $\mathrm{A}_{s}$ are the amplitudes of pump1, pump2, and the signal, respectively. The idler at a frequency of $\omega_{i}=\omega_{p 2}-\omega_{p 1}+\omega_{s}$ is generated by the resultant of two beating processes given by [38-40],

$$
\vec{E}_{i}=r\left(\omega_{p 2}-\omega_{p 1}\right)\left(\vec{E}_{p 2} \cdot \vec{E}_{p 1}^{*}\right) \vec{E}_{s}+r\left(\omega_{s}-\omega_{p 1}\right)\left(\vec{E}_{s} \cdot \vec{E}_{p 1}^{*}\right) \vec{E}_{p 2}
$$

where $r\left(\omega_{p 1}-\omega_{p 2}\right)$ is the efficiency of the beating process between the two pumps and $r\left(\omega_{s}-\omega_{p 1}\right)$ is the efficiency of the beating between the signal and the pump1 [41].

In the case of co-polarized pumps scheme, we tune the wavelengths of the two pumps such that the input signal is far enough from the pumps in order to avoid their interaction, which means $\left|\omega_{p 2}-\omega_{p 1}\right| \ll\left|\omega_{s}-\omega_{p 1}\right|$. Therefore, the electrical field of the idler can be given by,

$$
\vec{E}_{i}=r\left(\omega_{p 2}-\omega_{p 1}\right)\left(\vec{E}_{p 2} \cdot \vec{E}_{p 1}^{*}\right) \vec{E}_{s}
$$

Substituting Equation (5) to Equation (7) in Equation (9) gives,

$$
\vec{E}_{i}=r\left(\omega_{p 2}-\omega_{p 1}\right) \mathrm{A}_{p 1}^{*} \mathrm{~A}_{p 2} \vec{E}_{\mathrm{s}} \exp \left[j\left(\omega_{p 2}-\omega_{p 1}+\omega_{s}\right) t+\left(\phi_{p 2}-\phi_{p 1}+\phi_{s}\right)\right]
$$

It can be observed from Equation (10) that the generated idler has the same polarization as that of the input signal, and the output power is independent of the state of the polarization of the input 
signal. Assuming the SOA is polarization independent, the dual co-polarized pumping scheme can be used to convert a signal with arbitrary polarization with a constant conversion efficiency and single nonlinear SOA.

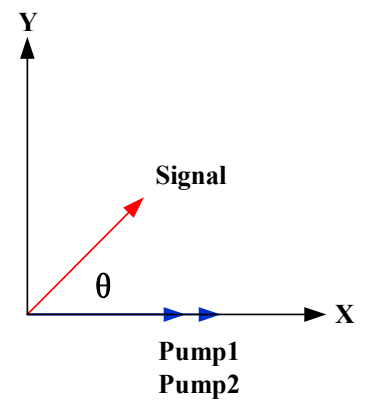

(a)

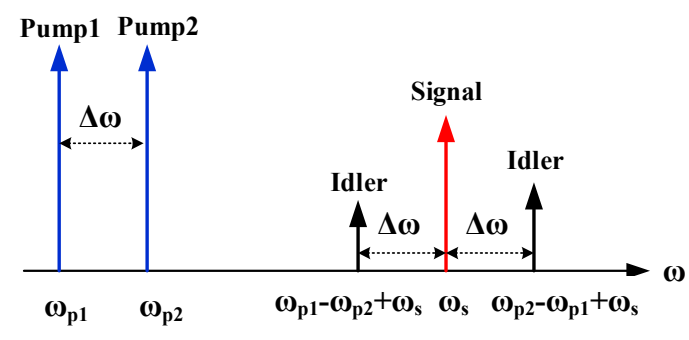

(b)

Figure 2. Four-wave mixing (FWM) scheme with dual co-polarized pumps. (a) The $X$ and $Y$ axes denote orthogonal states of polarization. In the dual pump FWM scheme, the polarization state of the wavelength-converted idler is that of the input signal provided both pumps have the same polarization.

(b) Spectral locations of the pumps, signal, and idlers.

\section{Linewidth Characterization of SGDBR Pump Lasers for Wavelength Conversion}

Among the tunable lasers, the SGDBR laser has been proven to be a suitable fast tunable laser candidate [42], and the structure of this type of laser is shown in Figure 3a. It is comprised of one gain section, one phase section, and two grating sections. Due to the period in which the two gratings are sampled differently, each grating has different period of reflection maxima. Therefore, the Vernier effect is enabled and enhances the overall tuning range considerably. The function of the phase section is to allow the cavity modes to be shifted independently of the grating's reflection peak, achieving continuous tuning. As the data is modulated on the phase of the laser in the transmission systems employing advanced modulation formats, the phase noise of the lasers becomes an important factor in determining the transmission performance [43]. For the SGDBR laser, both the gain section and the passive tuning sections can contribute to the overall phase noise of the laser [44]. In order to characterize the phase noise of the SGDBR laser, the frequency modulation (FM) noise spectrum, which has been proved to be a very suitable measurement of the phase noise of lasers to be employed in coherent systems [45], can be obtained by the technique using a coherent receiver with a narrow-linewidth LO [37]. As shown in Figure 3b, white noise from the gain section and low frequency carrier noise from the passive tuning sections are observed. Due to the advance in digital signal processing (DSP), the low frequency excess phase noise can be compensated. A 2nd-order PLL scheme has been used in the carrier phase estimation to track the excess phase noise [46], which presents the ability to employ fast tunable laser for higher order modulation formats.

Apart from that, the phase noise of the SGDBR laser is strongly dependent on the injection currents on each section, and the linewidth varies between several 100's $\mathrm{kHz}$ to several MHz with different injected currents. The output wavelength and phase noise of the SGDBR laser as a function of the injected currents in the front section are measured and shown in Figure 4, with $90 \mathrm{~mA}$ current on the gain section and $0 \mathrm{~mA}$ current on the phase and back section. The white noise and carrier noise are represented by the high frequency linewidth and low frequency linewidth, which are calculated from the FM-noise spectrum by the simple equation: $\Delta v=\pi \cdots S(f)$ [47]. The high frequency linewidth is related to the FM noise level in the frequency range beyond $500 \mathrm{MHz}$, whereas the low frequency linewidth contributed by the passive tuning sections is extracted by the mean value of the FM-noise in the frequency range under $50 \mathrm{MHz}$. It can be observed from Figure 4, by changing the currents into a grating section, that the entire reflection comb of the grating section shifts in wavelength and the laser wavelength jumps not only to an adjacent longitudinal mode, but can also jump by several 
$\mathrm{nm}$ to another super-mode at a wavelength where the reflection peaks of the Vernier-tuned gratings have re-aligned. By increasing the currents on the grating section, the low frequency linewidth and high frequency linewidth are found to present an opposite trend. The low frequency linewidth usually increases until the wavelength jump occurs, while the trend for the high frequency linewidth is decreased. As most low frequency phase noise can be compensated by the DSP, the operation points of the SGDBR laser with less high frequency noise are preferred to perform data transmission experiments. According to these linewidth measurements results, two wavelengths of the SGDBR laser are chosen to switch between for the later wavelength conversion experiment. The $1548.68 \mathrm{~nm}$ wavelength of the SGDBR, which is represented by the black point A shown in Figure 4, is chosen by applying $90 \mathrm{~mA}$ current on the gain section while the other passive tuning sections are terminated. The $1553.70 \mathrm{~nm}$ wavelength (point $\mathrm{B}$ ) is chosen by increasing the current on the front section to $3.4 \mathrm{~mA}$.
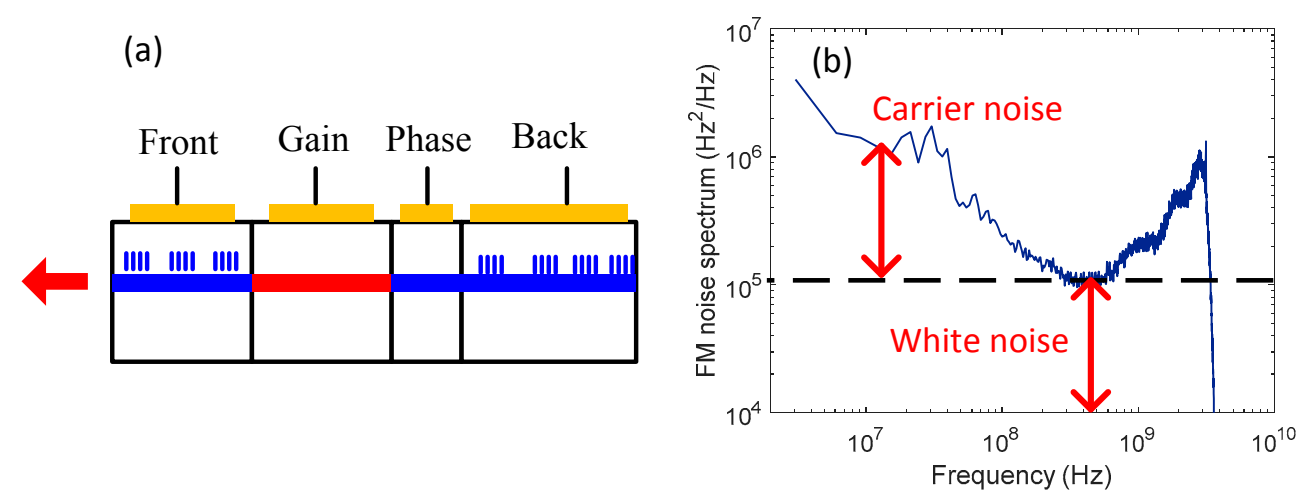

Figure 3. (a) Structure of the sampled, grating-distributed Bragg reflector (SGDBR) laser; (b) frequency modulation (FM) noise spectrum of the SGDBR laser.

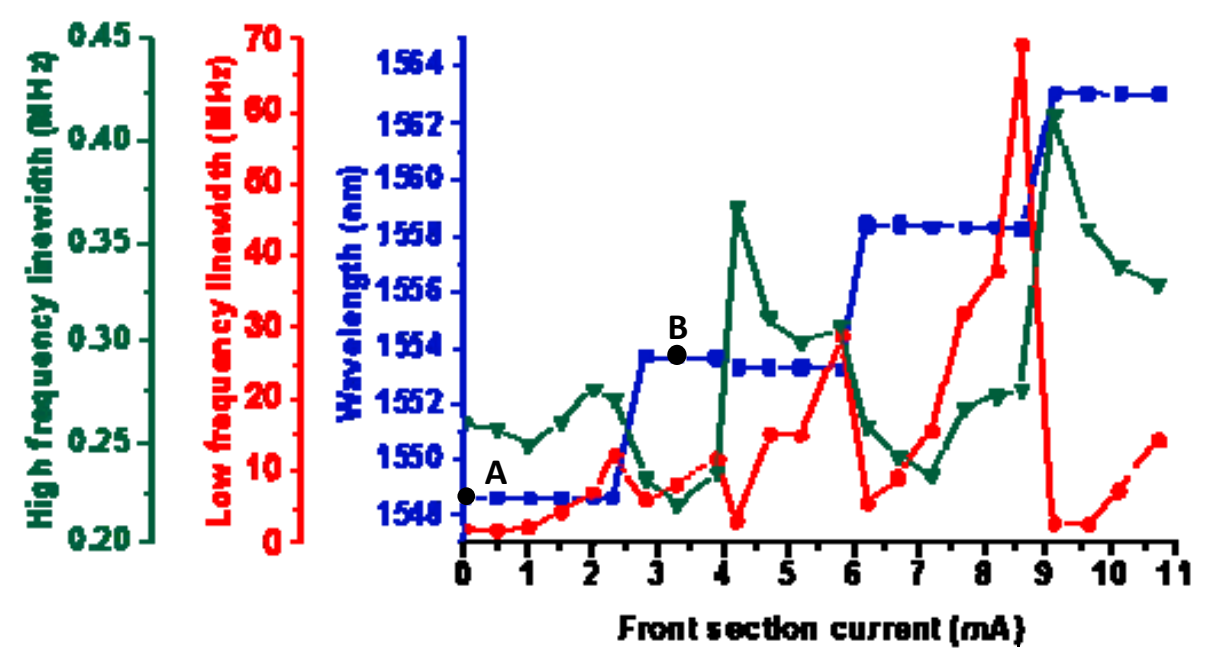

Figure 4. Measured high frequency linewidth (green triangles), low frequency linewidth (red dots), and output wavelength (blue squares) with different currents on the front section of the SGDBR laser, with $90 \mathrm{~mA}$ current on the gain section, $0 \mathrm{~mA}$ current on the phase and back section.

\section{Experimental Setup for Reconfigurable Wavelength Converter}

Figure 5 depicts the schematic diagram of the experimental setup of the SOA-based wavelength conversion of QPSK and PM-QPSK (blue dashed) signals using a SGDBR laser as one of the pump sources. A narrow linewidth external cavity laser (ECL) tuned to $1542.5 \mathrm{~nm}$ for all experiments is used as the signal source and was modulated with QPSK data at 12.5-Gbaud using an optical IQ modulator. The optical modulator is driven by electrical signals generated by the arbitrary waveform 
generator (AWG) with two uncorrelated pseudo-random bit sequences (PRBS) of $2^{7}-1$ bits periodicity. The AWG operated at $25 \mathrm{GSa} / \mathrm{s}$, which gives 2 samples per symbol for the 12.5-Gbaud QPSK signal. The two QPSK signals are amplified by using the radio frequency (RF) amplifiers. To implement the PM-QPSK signal scheme, the PM-QPSK signal is generated by using a Pol-Mux emulator, consisting of a polarization beam splitter (PBS) at its input, a passive stage with a delay of $4.88 \mathrm{~ns}$ (61 symbols), two polarization controllers, and a polarization beam combiner (PBC) to combine the polarization tributaries. The output of the two pumps are passed through polarization controllers, combined, and polarization-aligned using a $3 \mathrm{~dB}$ coupler and a PBS. For the wavelength conversion of the QPSK signal data, the generated optical QPSK signal is then coupled with two pumps (one SGDBR laser and one narrow linewidth ECL) and sent into the SOA-based wavelength converter. For both QPSK and PM-QPSK schemes, the power of the signal and the pumps at the input of the SOA are set at $-10 \mathrm{dBm}$ and $0 \mathrm{dBm}$, respectively, for optimum conversion efficiency [48]. The signal wavelength undergoes wavelength conversion through non-degenerate FWM in the SOA operating at $500 \mathrm{~mA}$ bias current. The SOA device used in the experiment is a nonlinear SOA with a very fast gain recovery time less than 25 ps. It operates over C-band with a typical small gain of $25 \mathrm{~dB}$, a saturation power of $+13 \mathrm{dBm}$, and less than $0.5 \mathrm{~dB}$ polarization-dependent saturated gain.

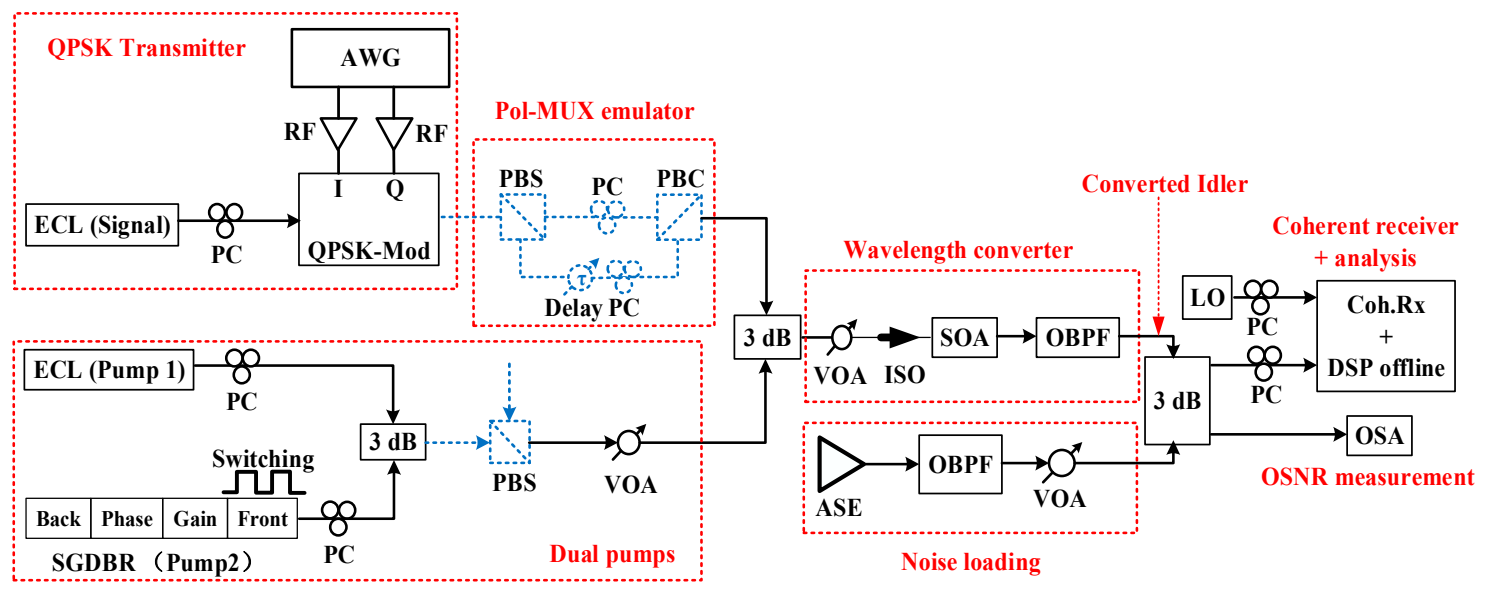

Figure 5. Schematic diagram of the reconfigurable SOA-based wavelength conversion of the quadrature phase shift keying (QPSK) and polarization multiplexed (Pol-Mux)-QPSK (blue dashed) signals employing a sampled grating distributed Bragg reflector (SGDBR) pump laser. PC: polarization controller, PBS: polarization beam splitter, PBC: polarization beam combiner, OBPF: optical band-pass filter, ISO: isolator, ASE: noise source, VOA: variable optical attenuator, OSA: optical spectrum analyzer, LO: local oscillator, Coh.Rx: coherent receiver.

After the FWM process in the SOA, the converted idler is then filtered out by using a tunable optical bandpass filter (OBPF). For system performance evaluation purposes, the optical signal to noise ratio (OSNR) of the idler is changed by adding amplified spontaneous emission (ASE) from an Erbium-doped fiber amplifier (EDFA) that is passed through a $2 \mathrm{~nm}$ bandwidth tunable optical bandpass filter. The filtered idler is then passed through a $3 \mathrm{~dB}$ splitter with one arm sent to the optical spectrum analyzer (OSA)for the OSNR measurement, and the other arm is passed into the polarization diversity coherent optical receiver and captured by a real-time oscilloscope sampling at $50 \mathrm{GSa} / \mathrm{s}$ for offline DSP processing. The received idler power at the input of the coherent receiver is maintained at $-19 \mathrm{dBm}$. The data captured from the real-time oscilloscope is first resampled to 2 samples per symbol using a priori knowledge of the clock frequency. Then the constant modulus algorithm (CMA) [49-51] is utilized to enable polarization de-multiplexing for PM-QPSK signal. An Mth power frequency offset compensation method [52-54] is employed to compensate the frequency offset between the converted idler signal and the local oscillator in the coherent receiver, with $m=4$ being the number of distinct 
phases in the QPSK symbol set. In order to make this frequency offset compensation method work correctly, it is essential to make sure that the absolute frequency offset is less than Rs/(2 M), where Rs is the symbol rate. A second order Phase-Locked Loop (PLL) is employed for the phase noise estimation [46], and the synchronization is achieved by adding training symbols at the beginning of the data [55] in order to carry out the BER calculation.

\section{Results and Discussion}

The wavelength of the fast-tuning SGDBR pump laser is switched between two operating modes by applying a switching signal to the wavelength tuning sections. In order to benchmark the system performance, we first present the BER performances when pump2 is tuned and fixed to the two wavelengths that pump2 will later be dynamically switched between. The wavelength and power of the signal and pump1 (ECL) are kept constant throughout. The input and output spectra of the SOA when the SGDBR laser is set at $1548.68 \mathrm{~nm}$ and $1553.70 \mathrm{~nm}$ are shown in Figure $6 \mathrm{a}, \mathrm{b}$, respectively, and it can be observed that the indicated idler of interest has changed wavelength position from Ch1 $(1541.395 \mathrm{~nm})$ to Ch2 (1538.684 nm).
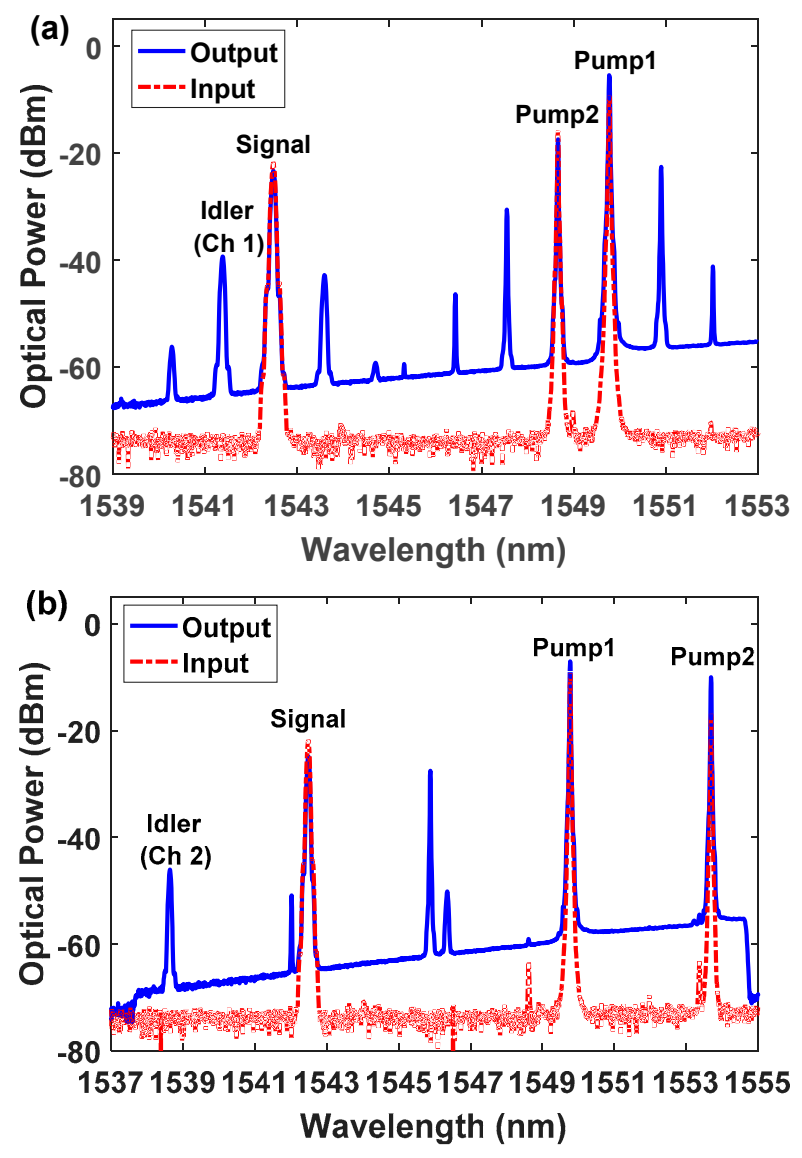

Figure 6. Input and output spectra of SOA showing the spectral locations of the signal of the external cavity laser (ECL), pump1 (ECL), pump2 (SGDBR), and converted idlers. (a) SOA input and output spectra when SGDBR is set at $1548.68 \mathrm{~nm}$. (b) SOA input and output spectra when SGDBR is set at $1553.70 \mathrm{~nm}$. The detected idlers are indicated.

BER performance as a function of OSNR at the receiver for the original signal; the signal after SOA and converted signals (idlers) for the QPSK and PM-QPSK signals at 12.5 GBaud are displayed in Figure $7 a, b$, respectively. It can be observed that the penalty between the original signal, the signal after SOA, and wavelength converted idler is under $0.5 \mathrm{~dB}$ for both cases, indicating the quality 
of the wavelength conversion scheme and potential usefulness. The constellation diagrams of the wavelength converted idler for a received OSNR of $12 \mathrm{~dB}$ and $14.5 \mathrm{~dB}$ are also given in Figure $7 \mathrm{a}, \mathrm{b}$, respectively. The phase noise of the FWM components is studied by using a coherent phase noise measurement technique [37,46]. The ECL's employed for the signal and pump1 have a linewidth of around $40 \mathrm{kHz}$ and the linewidth of the $1548.68 \mathrm{~nm}$ and $1553.70 \mathrm{~nm}$ wavelengths of the SGDBR laser (employed as the second pump for FWM scheme) are $260 \mathrm{kHz}$ and $230 \mathrm{kHz}$, respectively. The linewidth (from the high frequency phase noise region) of the idlers is measured to be around $370 \mathrm{kHz}$ and $300 \mathrm{kHz}$, as expected when the SGDBR is set to the two operating modes, since the idler linewidth is the sum of the linewidths of the pumps and signal, and in this case the linewidth of the SGDBR pump laser dominates.
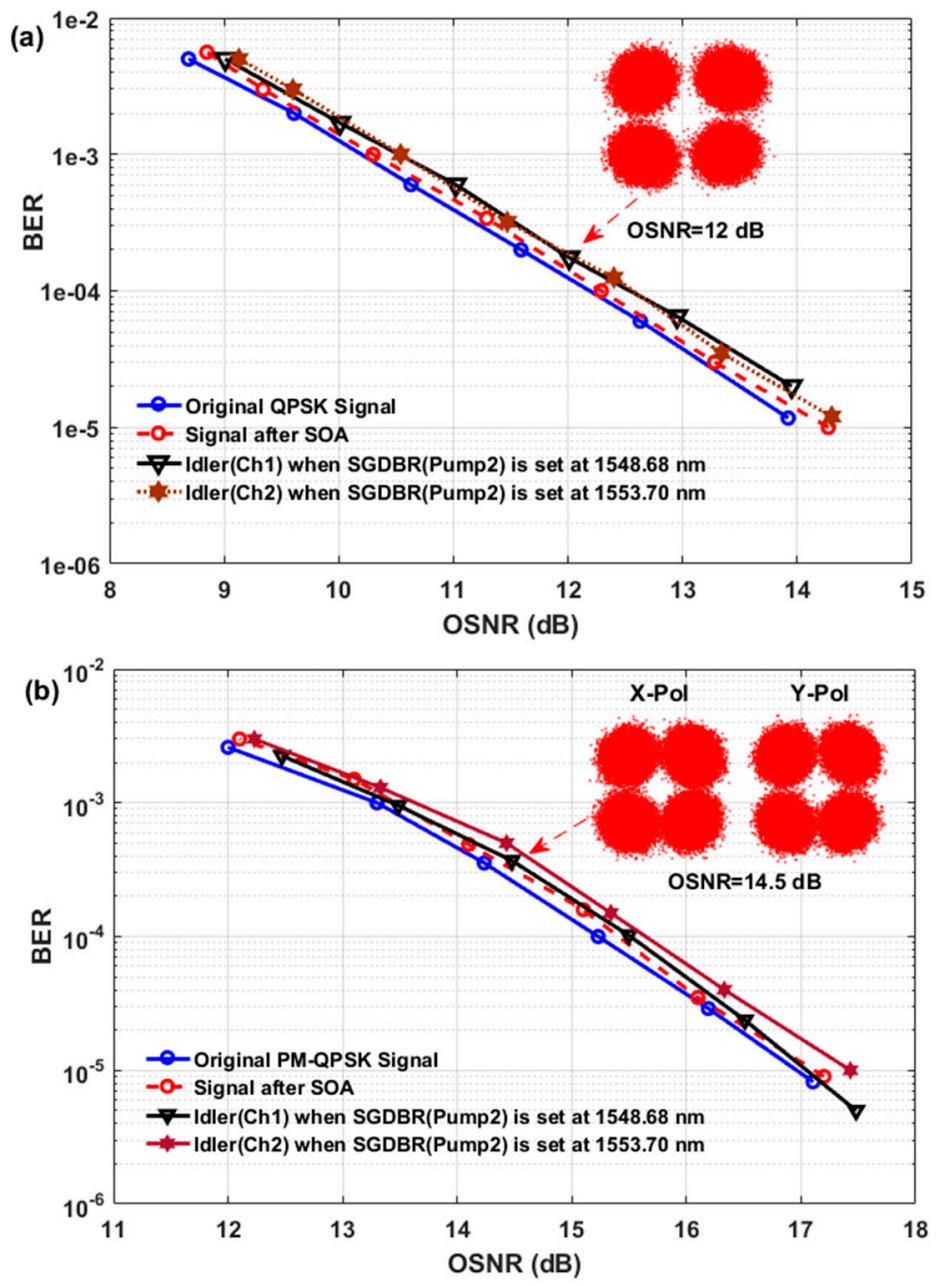

Figure 7. (a) Bit error rate (BER) versus optical signal to noise ratio (OSNR) curves for the input original signal, the signal after SOA, and the converted idlers for 12.5 GBaud QPSK signal. (b) BER versus OSNR curves for the input original signal, the signal after SOA, and the converted idlers for 12.5 GBaud PM-QPSK signal.

To calculate the limitations of the wavelength conversion scheme, we study the OSNR of the idler as a function of the detuning between the two pumps. The different wavelength converted idlers are filtered out by using an optical tunable band-pass filter. The OSNR measurement is then undertaken by using the OSA. In order to estimate the signal power and the noise floor correctly, the solution is to take two consecutive sweeps of the OSA with different resolution bandwidth (RBW) settings [56]. For the first sweep, OSA $0.2 \mathrm{~nm}$ RBW is used for the measurement of the signal power, 
and the second sweep measures the noise power by using $0.1 \mathrm{~nm}$ RBW setting. The output OSNR of the idler wavelength for the case with signal fixed at $1542.5 \mathrm{~nm}$, pump1 fixed at $1549.8 \mathrm{~nm}$, and the pump2 (SGDBR) tuned from $1548.7 \mathrm{~nm}$ to $1564.7 \mathrm{~nm}$ is displayed in Figure 8, which shows a tuning range of around $14 \mathrm{~nm}$ can be achieved with more than $9 \mathrm{~dB}$ OSNR, which is enough for the wavelength conversion of the QPSK data at 12.5 Gbaud to get a BER value below the $7 \%$ FEC limit $\left(3.8 \times 10^{-3}\right)$. Around $11 \mathrm{~nm}$ tuning range can be achieved for the wavelength conversion of PM-QPSK data to get a BER value below the $7 \%$ FEC limit. In order to investigate the time-resolved BER $[57,58]$ performance of the fast-reconfigurable wavelength converter, we apply a square wave current with $500 \mathrm{kHz}$ repetition rate to the front section of the SGDBR to switch the wavelength converted idler between Ch1 $(1541.395 \mathrm{~nm})$ and Ch2 $(1538.684 \mathrm{~nm})$, with the other currents applied to the SGDBR laser held constant and the received OSNR set at $12 \mathrm{~dB}$ for the QPSK signal and $14.5 \mathrm{~dB}$ for the PM-QPSK signal. The time-resolved BER measurement is characterized for $\mathrm{Ch} 1$ by fixing the center frequency of the OBPF to Ch1 and adjusting the $\mathrm{LO}$ to the wavelength of $\mathrm{Ch} 1$. The process is then repeated for $\mathrm{Ch} 2$.

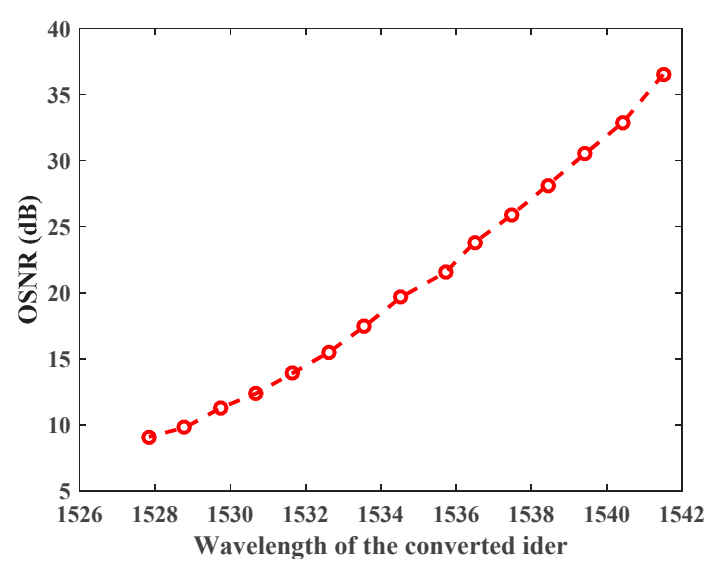

Figure 8. OSNR as a function of the wavelength of the converted idler.

We now present results of wavelength conversion when pump2 is dynamically tuned between $1547.68 \mathrm{~nm}$ and $1553.7 \mathrm{~nm}$. In order to accurately estimate the time-resolved BER performance after a switching event, a number of switching events are captured via multiple acquisitions using the real-time scope. Each data point in the time-resolved BER curves corresponds to the probability of receiving an error in a $10 \mathrm{~ns}$ period. This means the BER is averaged over a block length of 125 symbols (10 ns), and data captured from 200 switching events is used for the calculation, with a total of $5 \times 10^{4}$ bits and $10^{5}$ bits used for calculating each BER point in the time-resolved BER curves for QPSK and PM-QPSK signal at 12.5 GBaud, respectively. It can be seen from Figure $9 a, b$ that the reconfiguration time (time to have a BER better than the 7\% FEC limit) after a switch is approximately $50 \mathrm{~ns}$ and $160 \mathrm{~ns}$ for QPSK and PM-QPSK signal, respectively. The red curves in Figure 9a,b show the temporal frequency offset between the idler and the local oscillator in the coherent receiver after a switching event. It takes around $70 \mathrm{~ns}$ for the frequency of the wavelength converted idler for QPSK signal to fully stabilize after a switch, and $100 \mathrm{~ns}$ for PM-QPSK signal.

Figure 10 shows the BER measurement as a function of OSNR in a switching environment with different waiting time after the idler is switched to Ch1 and Ch2 when using QPSK and PM-QPSK decoding. The blue and red curves show the results for the data with a waiting time of $50 \mathrm{~ns}$ after switching when using QPSK decoding for Ch1 and Ch2, respectively. The brown and green curves are for a 160 ns waiting time when using PM-QPSK decoding for Ch1 and Ch2, respectively. The pink and black curves show the bad performance with a 50 ns waiting time using PM-QPSK decoding for $\mathrm{Ch} 1$ and $\mathrm{Ch} 2$, respectively. The required waiting time when using PM-QPSK decoding is longer than QPSK decoding mainly due to the longer convergence time associated with the CMA method used for de-multiplexing the dual-polarization packets. 

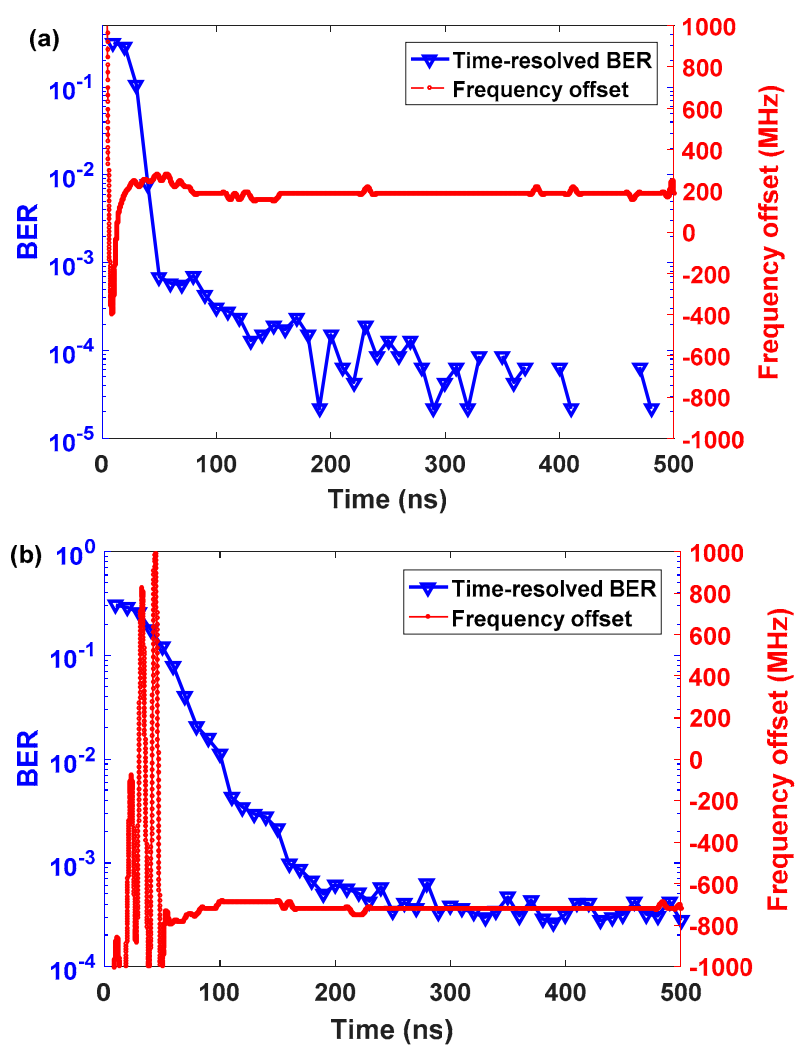

Figure 9. Time-resolved BER and frequency offset curves for: (a) QPSK signal; and (b) PM-QPSK signal at 12.5-GBaud, when the wavelength of the received signal (idler) is set at Ch1.

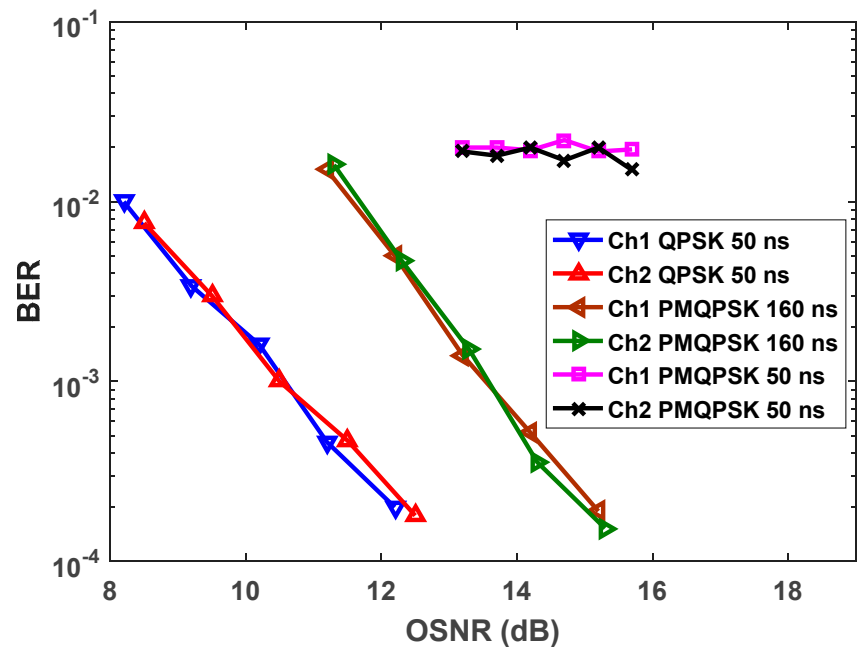

Figure 10. BER measurement as a function of OSNR in a switching environment with different waiting time after wavelength conversion when using QPSK and PM-QPSK decoding.

It also can be seen that the BER versus OSNR performance in a switching scenario corresponds with the static performance shown in Figure 7a,b, which indicates that the incoming signal can be precisely and quickly converted to the required channel on a timescale of around $50 \mathrm{~ns}$ and $160 \mathrm{~ns}$ for the 12.5-GBaud QPSK and PM-QPSK signal by using the wavelength converter we present. 


\section{Conclusions}

The wavelength conversion of data with advanced modulation formats will play a significant role in next generation optical networks. FWM is one of the most advantageous technologies for the wavelength conversion of data employing advanced modulation formats. We theoretically study the phase noise transfer issue for a nondegenerate FWM scheme and the vector theory in nonlinear SOAs. According to these phase noise and polarization studies, we design the efficient and impairment-free SOA-based wavelength converter based on FWM. We demonstrate a rapidly reconfigurable SOA-based FWM wavelength conversion system using a fast-switching, tunable SGDBR laser as one of the pumps and experimentally study the wavelength conversion of QPSK and Pol-Mul QPSK signals at 12.5-Gbaud, with total data rates of $25 \mathrm{Gbps}$ and $50 \mathrm{Gbps}$, respectively, using the proposed scheme. A wide tuning range $(>10 \mathrm{~nm})$ and a fast wavelength conversion time under $50 \mathrm{~ns}$ and $160 \mathrm{~ns}$ have been achieved for the proposed reconfigurable wavelength conversion system for QPSK and PM-QPSK signals, respectively. The reconfiguration time is mainly affected by the combination of the switching time of the tunable pump laser and the CMA convergence time in DSP. The performance under the switching environment after the required reconfiguration time is the same as the static case when the wavelengths are fixed, which makes it feasible to develop fast reconfigurable wavelength converters for dynamic, adaptive, and bandwidth-efficient optical networks by using rapid switching tunable pump lasers in conjunction with fast tuning optical filters.

Acknowledgments: This work has been supported by Science Foundation Ireland through grant numbers 13/RC/2077, 12/RC/2276, 15/US-C2C/I3132, and the HEA PRTLI 4 INSPIRE Programmes.

Author Contributions: L.P.B. conceived the initial ideas; Y.L., A.P.A., and F.L. designed and performed the experiments; Y.L., A.P.A., and S.P.Ó.D. wrote the paper. Y.Y. and L.P.B. reviewed the results and the manuscript.

Conflicts of Interest: The authors declare no conflict of interest.

\section{References}

1. Chang, G.K.; Yu, J.; Yeo, Y.K.; Chowdhury, A.; Jia, Z. Enabling technologies for next-generation optical packet-switching networks. Proc. IEEE 2006, 94, 892-910. [CrossRef]

2. Elmirghani, J.M.H.; Mouftah, H.T. All-optical wavelength conversion: Technologies and applications in DWDM networks. IEEE Commun. Mag. 2000, 38, 86-92. [CrossRef]

3. Bhopalwala, M.; Rastegarfar, H.; Kilper, D.C.; Wang, M.; Bergman, K. Energy efficiency of optical grooming of QAM optical transmission channels. Opt. Express 2016, 24, 2749-2764. [CrossRef] [PubMed]

4. Bayvel, P. Wavelength Routing and Optical Burst Switching in the Design of Future Optical Network Architectures; Proc. ECOC: Amsterdam, The Netherlands, 2001.

5. Govind, P. Agrawal, Population pulsations and nondegenerate four-wave mixing in semiconductor lasers and amplifiers. J. Opt. Soc. Am. B 1988, 5, 147-159.

6. Hu, H.; Jopson, R.M.; Gnauck, A.H.; Dinu, M.; Chandrasekhar, S.; Xie, C.; Randel, S. Parametric amplification wavelength conversion and phase conjugation of a 2.048- Tbit/s WDM PDM 16-QAM signal. J. Lightwave Technol. 2015, 33, 1286-1291. [CrossRef]

7. Li, C.; Luo, M.; He, Z.; Li, H.; Xu, J.; You, S.; Yang, Q.; Yu, S. Phase noise cancelled polarization-insensitive all-optical wavelength conversion of 557-Gb/s PDM-OFDM signal using coherent dual-pump. IEEE J. Lightwave Technol. 2015, 33, 2848-2854. [CrossRef]

8. Morshed, M.; Du, L.B.; Foo, B.; Pelusi, M.D.; Corcoran, B.; Lowery, A.J. Experimental demonstrations of dual polarization CO-OFDM using mid-span spectral inversion for nonlinearity compensation. Opt. Express 2014, 22, 10455-10466. [CrossRef] [PubMed]

9. Lu, G.; Albuquerque, A.; Puttnam, B.; Sakamoto, T.; Drummond, M.; Nogueira, R.; Kanno, A.; Shinada, S.; Wada, N.; Kawanishi, T. Pump-linewidth-tolerant optical wavelength conversion for high-order QAM signals using coherent pumps. Opt. Express 2014, 22, 5067-5075. [CrossRef] [PubMed]

10. Li, J.; O'Faolain, L.; Rey, I.H.; Krauss, T.F. Four-wave mixing in photonic crystal waveguides: Slow light enhancement and limitations. Opt. Express 2011, 19, 4458-4463. [CrossRef] [PubMed] 
11. Ettabib, M.A.; Lacava, C.; Liu, Z.; Bogris, A.; Kapsalis, A.; Brun, M.; Labeye, P.; Nicoletti, S.; Syvridis, D.; Richardson, D.J.; Petropoulos, P. Wavelength conversion of complex modulation formats in a compact SiGe waveguide. Opt. Express 2017, 25, 3252-3258. [CrossRef] [PubMed]

12. Adams, R.; Spasojevic, M.; Chagnon, M.; Malekiha, M.; Li, J.; Plant, D.V.; Chen, L.R. Wavelength conversion of 28 GBaud 16-QAM signals based on four-wave mixing in a silicon nanowire. Opt. Express 2014, 22, 4083-4090. [CrossRef] [PubMed]

13. D'Ottavi, A.; Iannone, A.; Mecozzi, A.; Scotti, S.; Spano, P.; Dall'Ara, R.; Eckner, J.; Guekos, G. Efficiency and noise performance of wavelength converters based on FWM in semiconductor optical amplifiers. IEEE Photonics Technol. Lett. 1995, 7, 357-359.

14. Contestabile, G.; Banchi, L.; Presi, M.; Ciaramella, E. Investigation of transparency of FWM in SOA to advanced modulation formats involving intensity, phase, and polarization multiplexing. J. Lightwave Technol. 2009, 27, 4256-4261. [CrossRef]

15. Kelly, A.E.; Ellis, A.D.; Nesset, D.; Kashyap, R. 100 Gbit/s wavelength conversion using FWM in an MQW semiconductor optical amplifier. Electron. Lett. 1998, 34, 1955-1956. [CrossRef]

16. Anthur, A.; Watts, R.T.; O'Carroll, J.; Venkitesh, D.; Barry, L.P. Dual correlated pumping scheme for phase noise preservation in all-optical wavelength conversion. Opt. Express 2013, 21, 15568-15579. [CrossRef] [PubMed]

17. Naimi, S.T.; Duill, S.P.O.; Barry, L.P. Detailed investigation of the pump phase noise tolerance for the wavelength conversion of 16-QAM signals using FWM. IEEE/OSA J. Opt. Commun. Netw. 2014, 6, 793-800. [CrossRef]

18. Dúill, S.P.Ó.; Naimi, S.T.; Anthur, A.P.; Huynh, T.N.; Venkitesh, D.; Barry, L.P. Simulations of an OSNR limited wavelength conversion scheme. IEEE Photonics Technol. Lett. 2013, 25, 2311-2314. [CrossRef]

19. Naimi, S.T.; Dúill, S.P.Ó.; Barry, L.P. Simulations of the OSNR and laser linewidth limits for reliable wavelength conversion of DQPSK signals using four-wave mixing. J. Opt. Commun. 2014, 310, 150-155. [CrossRef]

20. Dúill, S.P.Ó.; Naimi, S.T.; Anthur, A.P.; Huynh, T.N.; Venkitesh, D.; Barry, L.P. Numerical generation of laser-resonance phase noise for optical communication simulators. Appl. Opt. 2015, 54, 3398-3406. [CrossRef] [PubMed]

21. Anthur, A.P.; Watts, R.T.; Zhou, R.; Anandarajah, P.; Venkitesh, D.; Barry, L.P. Penalty-free wavelength conversion with variable channel separation using gain-switched comb source. Opt. Commun. 2015, 324, 69-72. [CrossRef]

22. Anthur, A.P.; Watts, R.T.; O'Duill, S.P.; Zhou, R.; Venkitesh, D.; Barry, L.P. Impact of nonlinear phase noise on all-optical wavelength conversion of 10.7 GBaud QPSK data using dual correlated pumps. IEEE J. Quantum Electron. 2015, 51, 9100105. [CrossRef]

23. Filion, B.; Ng, W.C.; Nguyen, A.T.; Rusch, L.A.; LaRochelle, S. Wideband wavelength conversion of 16 Gbaud 16-QAM and 5 Gbaud 64-QAM signals in a semiconductor optical amplifier. Opt. Express 2013, 21, 19825-19833. [CrossRef] [PubMed]

24. Contestabile, G.; Yoshida, Y.; Maruta, A.; Kitayama, K. Coherent wavelength conversion in a quantum dot SOA. IEEE Photonics Technol. Lett. 2013, 25, 791-794. [CrossRef]

25. Krzczanowicz, L.; Connelly, M.J. 40 Gb/s NRZ-DQPSK data all-optical wavelength conversion using four wave mixing in a bulk SOA. IEEE Photonics Technol. Lett. 2013, 25, 2439-2441. [CrossRef]

26. Naimi, S.T.; Duill, S.P.Ó;; Barry, L.P. All Optical Wavelength Conversion of Nyquist-WDM Superchannels Using FWM in SOAs. J. Lightwave Technol. 2015, 33, 3959-3967. [CrossRef]

27. Dúill, S.P.Ó.; Barry, L.P. Improved reduced models for single-pass and reflective semiconductor optical amplifiers. J. Opt. Commun. 2015, 334, 170-173. [CrossRef]

28. Inoue, K. Polarization effect on four-wave mixing efficiency in a single-mode fiber. IEEE J. Quantum Electron. 1992, 28, 883-894. [CrossRef]

29. Anthur, A.P.; Zhou, R.; O’Duill, S.; Walsh, A.J.; Martin, E.; Venkitesh, D.; Barry, L.P. Polarization insensitive all-optical wavelength conversion of polarization multiplexed signals using co-polarized pumps. Opt. Express 2016, 24, 11749-11761. [CrossRef] [PubMed]

30. Hui, R.; Mecozzi, A. Phase noise of four-wave mixing in semiconductor lasers. Appl. Phys. Lett. 1992, 60, 2454-2456. [CrossRef] 
31. Blumenthal, D.J.; Bowers, J.E.; Rau, L.; Hsu-Feng, C.; Rangarajan, S.; Wei, W.; Poulsen, K.N. Optical signal processing for optical packet switching networks. IEEE Commun. Mag. 2003, 41, S23-S29. [CrossRef]

32. Sadot, D.; Boimovich, E. Tunable optical filters for dense WDM networks. IEEE Commun. Mag. 1998, 36, 50-55. [CrossRef]

33. Jayaraman, V.; Mathur, A.; Coldren, L.A.; Dapkus, P.D. Theory design and performance of extended tuning range in sampled grating DBR lasers. IEEE J. Quantum Electron. 1993, 29, 1824-1834. [CrossRef]

34. Ward, A.J.; Robbins, D.J.; Busico, G.; Barton, E.; Ponnampalam, L.; Duck, J.P.; Whitbread, N.D.; Williams, P.J.; Reid, D.C.J.; Carter, A.C.; et al. Widely tunable DS-DBR laser with monolithically integrated SOA: Design and performance. IEEE J. Sel. Top. Quantum Electron. 2005, 11, 149-156. [CrossRef]

35. Wesström, J.-O.; Hammerfeldt, S.; Buus, J.; Siljan, R.; Laroy, R.; de Vries, H. Design of a widely tunable modulated grating Y-branch laser using the additive Vernier effect for improved super-mode selection. In Proceedings of the 18th International Semiconductor Laser Conference (ISLC), Garmisch, Germany, 29 September-3 October 2002.

36. Lin, Y.; Anthur, A.P.; O'uill, S.; Naimi, S.T.; Yu, Y.; Barry, L. Fast Reconfigurable SOA-Based All-Optical Wavelength Conversion of QPSK Data Employing Switching Tunable Pump Lasers; Proc. OFC: Los Angeles, CA, USA, 2017.

37. Kikuchi, K. Characterization of semiconductor-laser phase noise and estimation of bit-error rate performance with low-speed offline digital coherent receivers. Opt. Express 2012, 20, 5291-5302. [CrossRef] [PubMed]

38. Kyo, I. Polarization independent wavelength conversion using fiber four-wave mixing with two orthogonal pump lights of different frequencies. J. Lightwave Technol. 1994, 12, 1916-1920.

39. Jia, L.; Chen, L.; Dong, Z.; Cao, Z.; Wen, S. Polarization insensitive wavelength conversion based on orthogonal pump four-wave mixing for polarization multiplexing signal in high-nonlinear fiber. J. Lightwave Technol. 2009, 27, 5767-5774. [CrossRef]

40. Jonathan, L.; Mark, P.R.; Summerfield, A.; Madden, S.J. Tunability of polarization-insensitive wavelength converters based on four-wave mixing in semiconductor optical amplifiers. J. Lightwave Technol. 1998, 16, 2419-2427.

41. Zhou, J.; Park, N.; Vahala, K.J.; Newkirk, M.; Miller, B.I. Four-wave mixing wavelength conversion efficiency in semiconductor traveling-wave amplifiers measured to $65 \mathrm{~nm}$ of wavelength shift. IEEE Photonics Technol. Lett. 1994, 6, 984-987. [CrossRef]

42. Delorme, F. Widely tunable $1.55 \mu \mathrm{m}$ lasers for wavelength-division-multiplexed optical fiber communications. IEEE J. Quantum Electron. 1998, 34, 1706-1716. [CrossRef]

43. Seimetz, M. Laser Linewidth Limitations for Optical Systems with High-Order Modulation Employing Feed forward Digital Carrier Phase Estimation; Proc. OFC: San Diego, CA, USA, 2008.

44. Jialin, Z.; Huijuan, Z.; Fan, L.; Yonglin, Y. Numerical Analysis of Phase Noise Characteristics of SGDBR Lasers. J. Sel. Top. Quantum Electron. 2015, 21, 1502009. [CrossRef]

45. Camatel, S.; Ferrero, V. Narrow linewidth CW laser phase noise characterization methods for coherent transmission system applications. J. Lightwave Technol. 2008, 26, 3048-3054. [CrossRef]

46. Huynh, T.N.; Nguyen, A.T.; Ng, W.C.; Nguyen, L.; Rusch, L.A.; Barry, L.P. BER Performance of Coherent Optical Communications Systems Employing Monolithic Tunable Lasers With Excess Phase Noise. J. Lightwave Technol. 2014, 32, 1973-1980. [CrossRef]

47. Di Domenico, G.; Schilt, S.; Thomann, P. Simple approach to the relation between laser frequency noise and laser line shape. Appl. Opt. 2010, 49, 4801-4807. [CrossRef] [PubMed]

48. Baveja, P.P.; Maywar, D.N.; Agrawal, G.P. Interband four-wave mixing in semiconductor optical amplifiers with ASE-enhanced gain recovery. IEEE J. Sel. Top. Quantum Electron. 2012, 18, 899-908. [CrossRef]

49. Liu, L.; Tao, Z.; Yan, W.; Oda, S.; Hoshida, T.; Rasmussen, J.C. Initial Tap Setup of Constant Modulus Algorithm for Polarization De-Multiplexing in Optical Coherent Receivers; Proc. OFC: San Diego, CA, USA, 2009.

50. Kikuchi, K. Performance analyses of polarization demultiplexing based on constant-modulus algorithm in digital coherent optical receivers. Opt. Express 2011, 19, 9868-9980. [CrossRef] [PubMed]

51. Leven, A.; Kaneda, N.; Chen, Y. A Real-Time CMA-Based 10 Gb/s Polarization Demultiplexing Coherent Receiver Implemented in an FPGA; Proc. OFC: San Diego, CA, USA, 2008.

52. Maher, R.; Millar, D.S.; Savory, S.J.; Thomsen, B.C. Widely Tunable Burst Mode Digital Coherent Receiver With Fast Reconfiguration Time for 112 Gb/s DP-QPSK WDM Networks. J. Lightwave Technol. 2012, 30, 3924-3930. [CrossRef] 
53. Simsarian, J.E.; Gripp, J.; Gnauck, A.H.; Raybon, G.; Winzer, P.J. FastTuning 224-Gb/s Intradyne Receiver for Optical Packet Networks; Proc. OFC: San Diego, CA, USA, 2010.

54. Nakagawa, T.; Matsui, M.; Kobayashi, T.; Ishihara, K.; Kudo, R.; Mizoguchi, M.; Miyamoto, Y. Non-Data-Aided Wide-Range Frequency Offset Estimator for QAM Optical Coherent Receivers; Proc. OFC: Los Angeles, CA, USA, 2011.

55. Mori, Y.; Zhang, C.; Igarashi, K.; Katoh, K.; Kikuchi, K. Unrepeated 200-km transmission of 40-Gbit/s 16-QAM signals using digital coherent receiver. Opt. Express 2009, 17, 1435-1441. [CrossRef] [PubMed]

56. Dupre, J.; Stimple, J. Making OSNR Measurements in a Modulated DWDM Signal Environment. Available online: http:/ / www.keysight.com/upload/cmc_upload/All/SLDPRE_2_OSNR_Measure.pdf (accessed on 8 October 2017).

57. O’Dowd, J.A.; Shi, K.; Walsh, A.J.; Bessler, V.M.; Smyth, F.; Huynh, T.N.; Barry, L.P.; Ellis, A.D. Time resolved bit error rate analysis of a fast switching tunable laser for use in optically switched networks. J. Opt. Commun. Netw. 2012, 4, A77-A81. [CrossRef]

58. Smyth, F.; Browning, C.; Shi, K.; Peters, F.; Corbett, B.; Roycroft, B.; Barry, L.P. 10.7Gbd DQPSK Packet Transmission Using a Widely Tunable Slotted Fabry-Perot Laser; Proc. ECOC: Torino, Italy, 2010.

(C) 2017 by the authors. Licensee MDPI, Basel, Switzerland. This article is an open access article distributed under the terms and conditions of the Creative Commons Attribution (CC BY) license (http:/ / creativecommons.org/licenses/by/4.0/). 